\title{
Brief, non-directive psychotherapy and GP care both improved patients' emotional difficulties
}

Friedli K, King MB, Lloyd M, et al. Randomised controlled assessment of non-directive psychotherapy versus routine general-practitioner care. Lancet 1997 Dec 6;350:1662-5.

\section{Question}

In patients with emotional difficulties is brief, non-directive psychotherapy more effective than routine general practitioner (GP) care?

\section{Design}

Randomised controlled trial with 9 months follow up.

\section{Setting}

14 practices in north west London, UK.

\section{Patients}

136 patients who were $\geqslant 18$ years of age (mean age $39 \mathrm{y}, 81 \%$ women) and had emotional difficulties starting within the previous 6 months that their GP felt required a brief, non-directive psychotherapy intervention. Exclusion criteria were current receipt of brief psychotherapy or other form of psychological intervention, psychotic or chronic mental illness, suicidal tendencies, severe drug or alcohol dependency, physical illness precluding coming to the doctor's office, or inability to complete questionnaires. Follow up at 9 months was $86 \%$.

\section{Intervention}

Patients were allocated to brief, non-directive psychotherapy $(n=70)$ or routine care from their GP $(n=66)$. 4 general practice based therapists employed for the study used the Rogerian model of psychotherapy that involved helping the patient express feelings, clarify thoughts, and restate or reframe difficulties using empathy and advanced listening skills. Patients who were allocated to GP care discussed their difficulties as they would do in normal practice. GPs were discouraged from referring patients to a therapist.

\section{Main outcome measures}

Psychological outcomes were measured by the Beck Depression Inventory (BDI), the Brief Symptom Inventory (BSI), and the
Revised Clinical Interview Schedule (CIS). Social outcome was measured by the Modified Social Adjustment Scale. Patient satisfaction was assessed by questionnaire.

\begin{abstract}
Main results
Analysis was by intention to treat and controlled for baseline scores. The study had $80 \%$ power with 64 patients in each group to detect a treatment effect of 0.5 for brief, non-directive psychotherapy on BDI scores. All outcome measures decreased in severity over time and did not differ between groups at 3 months or at 9 months $(\mathrm{p}>0.10)$. The table shows mean scores at 9 months for patients who received brief psychotherapy compared with GP care. Patients were more satisfied with the therapist than with the GP (mean difference in total score on the satisfaction questionnaire at 9 months $8.5,95 \%$ CI 4.1 to 12.7 ).
\end{abstract}

\section{Conclusion}

In patients with emotional difficulties, care provided by general practitioners and brief, non-directive psychotherapy were equally effective but patients were more satisfied with the care they received from psychotherapists.

Difference in mean scores at 9 months for brief psychotherapy v GP care

\begin{tabular}{lcc}
\hline Outcomes & Brief psychotherapy & GP care \\
\hline Beck depression inventory & 9.7 & 13.5 \\
Brief symptom inventory & 0.7 & 0.9 \\
Revised clinical interview schedule & 12.2 & 13.5 \\
Modified social adjustment scale & 2.0 & 2.2 \\
\hline
\end{tabular}

Sources of funding: Mental Health Foundation; Medical Research Council; (former) North East Thames Regional Health Authority.

For correspondence: Professor M B King, University Department of Psychiatry, Royal Free Hospital School of Medicine, London NW3 2PF, UK. Fax +44 (O)171 8302808.

\section{Commentary}

In the UK, patients only attend the particular GP with whom they are registered, and the GP initiates all specialist referrals, including those for non-directive psychotherapy. In this study by Friedli $e t a l$, the study sample should be representative of people seeking help who have emotional problems, although as the authors point out, GPs may have been selective in choosing which patients to enroll. Inclusion criteria were broad, chosen to reflect the way GPs make management decisions in routine practice, and hence were appropriate to the research question. However, the resulting sample heterogeneity complicates the choice of suitable outcome measures. The measured outcomes cannot confidently be attributed to the interventions for 2 reasons. Firstly, although the authors cite evidence indicating that psychiatric problems in primary care may endure, other data indicate that even among patients with more enduring psychological problems than those in this study, some get better without specific interventions. ${ }^{1}$ Secondly, although the BSI has been used before in non-directive psychotherapy outcome studies, the CIS is designed to measure psychiatric symptoms rather than "emotional difficulties," and because psychotherapy did not aim directly to reduce psychopathology, it may be that the CIS was not sufficiently sensitive to measure changes resulting from psychotherapy. Conversely, the patient satisfaction questionnaire results could be explained if this questionnaire better reflected what the therapists did rather than what the GPs did. This illustrates a recurrent conundrum for primary care researchers. If inclusion criteria are made more specific, results are less likely to be generalisable, whereas if they are kept broad, valid outcome measures are much harder to define. This study has made what is probably one of the best attempts to date to solve this dilemma in the context of non-directive psychotherapy in primary care. Overall, the results fail to support the value of non-directive psychotherapy, although patients were satisfied with this (and probably GPs were also). Ironically, in a post hoc subgroup analysis, non-directive psychotherapy benefited patients who were clinically depressed, for whom there is already good evidence for the efficacy of other psychological interventions, notably cognitive behavioural therapy and interpersonal therapy.

Tom Sensky, PhD, MB, FRCPsych Imperial College School of Medicine West Middlesex University Hospital Isleworth, Middlesex, UK Brodaty H, Andrews G. Br J Psychiatry
1983;143:11-9. 